\title{
THE VARIATION OF THE SIGN OF THE REAL PART OF A MEROMORPHIC FUNCTION ON THE UNIT CIRCLE
}

\author{
BY \\ JUN-ITI ITO
}

1. Introduction. The purpose of this paper is to prove the two theorems on functions meromorphic in $|z| \leqq 1$ and having zeros and poles on $|z|=1$. One theorem is a generalization of the result obtained by the author [7], for the meromorphic function in $|z| \leqq 1$ with assigned zeros and poles in $|z|<1$ and no zero and pole on $|z|=1$, which was a generalization of the results proved by S. Kakeya [8] and the author [6], and the other is a new attempt to generalize the results obtained by Goodman and Robertson [4] and Robertson [13]. As corollaries and applications of these theorems we give some precisions and generalizations of the results obtained by Goodman [3], Goodman and Robertson [4] and Robertson [13].

\section{Preliminary.}

Definition 1. The real part of a meromorphic function $f(z)$ on $|z|=1$ is said to change sign $2 p$ times on $|z|=1$ and $f(z)$ is said to belong to the class $M(p)$ if there are $2 p$ values of $\theta,-\pi \leqq \tau_{1}<\cdots<\tau_{2 p}<\pi$, such that, $\varepsilon$ being +1 or -1 ,

$$
\begin{aligned}
& \mathcal{E R f}\left(e^{i \theta}\right) \begin{cases}\geqq 0 \text { for } \tau_{2 m}>\theta>\tau_{2 m-1}, & m=1,2, \cdots, p, \\
\leqq 0 \text { for } \tau_{2 m+1}>\theta>\tau_{2 m}, & m=1,2, \cdots, p-1,\end{cases} \\
& \tau_{1}>\theta \geqq-\pi, \pi>\theta>\tau_{2 p}
\end{aligned}
$$

except for the poles on $|z|=1$.

In particular, if there exists a $\lambda>0$ for each $e^{i \tau_{\Sigma}}, K=1, \cdots, 2 p$, such that for $0<\delta<\lambda$,

$$
\begin{aligned}
& R f\left(e^{i\left(\tau_{K}+\delta\right)}\right) R f\left(e^{i\left(\tau_{K}-\delta\right)}\right)<0 \text { for }\left|\tau_{K}\right|<\pi, \\
& R f\left(e^{i(\pi-\delta)}\right) R f\left(e^{-i(\pi-\delta)}\right)<0 \text { for } \tau_{1}=-\pi,
\end{aligned}
$$

$R f(z)$ is said to change sign exactly $2 p$ times on $|z|=1$, and $f(z)$ is said to belong the class $M^{\prime}(p)$.

DEFINITION 2. Any point $e^{i \zeta_{K}},-\pi \leqq \zeta_{K}<\pi$, is said to be the negative point if

(a) $e^{i \zeta_{K}}$ is a point among $e^{i \tau_{K}}, K=1, \cdots, 2 p$, except for the poles,

(b) $\varepsilon(-1)^{K} I f\left(e^{i \zeta_{K}}\right) \leqq 0$.

Any point of $e^{i_{\tau_{K}}}, K=1, \cdots, 2 p$, distinct from the negative points is said to be the positive point.

Received by the editors March 17, 1955 and, in revised form, February 15, 1957. 
Definition 3. A function $f(z)$ is said to belong to the class $R_{1}(p, m)$ where $p$ and $m$ are non-negative integers if

(a) $f(z)$ is meromorphic on $|z|=1$,

(b) $R f(z)$ changes sign $2 p$ times on $|z|=1$,

(c) the number of the positive points is $m$.

Definition 4. A function $f(z)$ is said to belong to the class $R_{\mathbf{2}}(p, m)$ if

(a) $f(z)$ is a function of the class $R_{1}(p, m)$,

(b) any positive point $e^{i \tau \pi}$ satisfies the following two conditions such that for $r<1$,

(1) $e^{i \tau K}$ is at most a 2 nd order pole,

(2) $\lim _{r \rightarrow 1}(-1)^{K} \mathcal{E}(1-r)^{2} I f\left(r e^{i \tau K}\right) \geqq 0$,

(c) any pole $e^{i \zeta},|\zeta|<\pi$, distinct from $e^{i \tau K}, K_{\varepsilon_{i}}, \cdots, 2 p$, satisfies the two conditions such that for $r<1$,

(1) $e^{i s}$ is a first order pole,

$$
\begin{aligned}
& \lim _{r \rightarrow 1} \mathcal{E}(1-r) R f\left(r e^{i \zeta}\right) \begin{cases}\geqq 0 \text { for } \tau_{2 m}>\zeta>\tau_{2 m-1}, & m=1, \cdots, p, \\
\leqq 0 \text { for } \tau_{2 m+1}>\zeta>\tau_{2 m}, & m=1, \cdots, p-1,\end{cases} \\
& \tau_{1}>\zeta \geqq-\pi, \pi>\zeta>\tau_{2 p} \text {. }
\end{aligned}
$$

For a function of the class $M(p)$, we define the generalized order of the zeros or poles on $|z|=1$ as follows:

(A) If $q$ is an arbitrary odd number and if a $q$ th order zero or pole $e^{i 5}$, $|\zeta|<\pi$, on $|z|=1$ distinct from $e^{i \tau K}, K=1, \cdots, 2 p$, satisfies the two conditions

$\lim (-1)^{[q / 2]} \mathcal{E}(1-r)^{q} R\left\{f\left(r e^{i \zeta}\right)^{ \pm 1}\right\}$

$$
\begin{aligned}
& \cdot \begin{cases}<0 \text { for } \tau_{2 m}>\zeta>\tau_{2 m-1}, & m=1, \cdots, p \\
>0 \text { for } \tau_{2 m+1}>\zeta>\tau_{2 m}, & m=1, \cdots, p-1,\end{cases} \\
& \tau_{1}>\zeta \geqq-\pi, \pi>\zeta>\tau_{2 p}
\end{aligned}
$$

where the double sign denotes - when $e^{i 5}$ zero and + when pole, then the generalized order of the above zero or pole on $|z|=1$ is defined by $q+1$ respectively.

(B) If $q$ is an arbitrary even number and if a $q$ th order zero or pole $e^{i \zeta_{K}}$ among $e^{i \tau K}, K=1, \cdots, 2 p$, satisfies

$$
\lim _{r \rightarrow 1}(-1)^{K+q / 2} \mathcal{E}(1-r)^{q} I\left\{f\left(r e^{i \zeta K}\right)^{ \pm 1}\right\}<0
$$

where the double sign obeys the rule in (A), the generalized order of this zero or pole is defined by $q-2$ respectively.

(C) The generalized order of any $q$ th, $q \geqq 1$, order zero or pole on $|z|=1$ except for the case of (A) and (B) is defined by $2[q / 2]$.

Definition 5. All the zeros or poles on $|z|=1$ of which the multiplicities 
are defined by its generalized order are said to be closed zero or poles respectively.

Lemma 1. Let $f(z)$ be a member of the class $R_{1}(p, m)$. Denote the closed zeros and poles of $f(z)$ be $e^{i \delta 2 p-m+K}, K=1, \cdots, 2 s_{1}$, and $e^{i \sigma m+K}, K=1, \cdots, 2 t_{1}$, respectively. Suppose that $F_{1}(z)$ is defined by $\left.{ }^{1}\right)$

$$
\begin{aligned}
F_{1}(z)= & (-1)^{s 1-t_{1}} z^{s 1-t_{1}} \exp \left\{\frac{i}{2}\left(\sum_{K=1}^{2 s_{1}} \delta_{2 p-m+K}-\sum_{K=1}^{2 t_{1}} \sigma_{m+K}\right)\right\} \\
& \cdot \prod_{K=1}^{2 t_{1}}\left(e^{i \sigma_{m}+k}-z\right) \prod_{K=1}^{2 s_{1}}\left(e^{i \delta_{2 p-m}+K}-z\right)^{-1} .
\end{aligned}
$$

Then $F_{1}(z) f(z)$ and $\left\{F_{1}(z) f(z)\right\}^{-1}$ are members of the classes $R_{2}(p, m)$ and $R_{2}(p, 2 p-m)$ respectively.

Proof. From the definition of the closed zeros and poles on $|z|=1$, the generalized order of these zeros and poles are all even. Therefore $F_{1}(z)$ in (2.1) can be written in the following form:

$$
F_{1}(z)=z^{s_{1}-t_{1}} \prod_{K=1}^{t_{1}}\left(z-e^{i \beta_{K}}\right)\left(1-e^{-i \beta_{K}} z\right) \prod_{K=1}^{s_{1}}\left(z-e^{i \alpha_{K}}\right)^{-1}\left(1-e^{-i \alpha_{K} z}\right)^{-1}
$$

So that by the definitions (A), (B) and (C), both $F_{1}(z) f(z)$ and $\left\{F_{1}(z) f(z)\right\}^{-1}$ satisfy the Conditions (b) and (c) in Definition 4. Thus the proof is completed.

Lemma 2. Let $f(z)$ be meromorphic for $|z| \leqq 1$ and $f(z)$ a member of the class $R_{2}(p, m)$. Denote the zeros and poles of $f(z)$ in $|z|<1$ by $b_{1}, \cdots, b_{s}$ and $d_{1}, \cdots, d_{t}$ respectively. Further, let $\left({ }^{2}\right)$

$$
F_{2}(z)=z^{s-t} \prod_{K=1}^{t}\left(z-d_{K}\right)\left(1-\bar{d}_{K} z\right) \prod_{K=1}^{s}\left(z-b_{K}\right)^{-1}\left(1-b_{K} z\right)^{-1} .
$$

Then $F_{2}(z) f(z)$ is a member of the class $R_{2}(p, m)$.

This lemma is easily proved.

Lemma 3. Let a function $f(z)$ be a member of the class $R_{2}(p, m)$. Denote the positive and negative points of $f(z)$ by $e^{i \sigma_{1}}, \cdots, e^{i \sigma_{m}}$ such that $-\pi \leqq\left|\sigma_{K}\right|<\pi$, $K=1, \cdots, m$, and $e^{i \delta_{1}}, \cdots, e^{i \delta 2 p-m}$ such that $-\pi \leqq\left|\delta_{K}\right|<\pi, K=1, \cdots$, $2 p-m$, respectively. Consider the function

$$
\begin{aligned}
& g(z)=(-1)^{p-m-1} \exp \left\{\frac{i}{2}\left(\sum_{K=1}^{2 p-m} \delta_{K}-\sum_{K=1}^{m} \sigma_{K}\right)\right\} \\
& z^{p-m} \prod_{K=1}^{m}\left(e^{i \sigma_{K}}-z\right) \prod_{K=1}^{2 p-m}\left(e^{i \delta_{K}}-z\right)^{-1} .
\end{aligned}
$$

(1) Here and in the rest of the paper a $q$ th generalized order of the closed zero appears $q$ times in $e^{i \alpha_{1}}, \cdots, e^{i \alpha_{2 s}}$, and $2 s$ is the number of the closed zeros $e^{i \alpha}$ counted in accordance with their multiplicities. For the closed pole the above rules are also to be taken.

(2) Functions $F_{2}(z)$ and $g(z)$ were used in the previous paper [7]. 
Then $F(z)=g(z) f(z)$ is a member of the class $R_{2}(0,0)$.

Proof. By Definition 1, for each $\theta,-\pi \leqq \theta<\pi, R F\left(e^{i \theta}\right) \geqq 0$ except for the poles of $F(z)$ on $|z|=1$. By Definition $4, F(z)$ has at most first order poles on $|z|=1$, and for each positive points $e^{i \sigma K}, K=1, \cdots, m$,

$$
\lim _{r \rightarrow 1} \mathcal{E}(1-r) R F\left(r e^{i \sigma_{K}}\right)
$$

$$
\begin{aligned}
& =-2^{2(m-p)+1} \prod_{i(\neq K)=1}^{m} \sin \frac{\sigma_{i}-\sigma_{K}}{2} \prod_{j=1}^{2 p-m}\left(\sin \frac{\delta_{j}-\sigma_{K}}{2}\right)^{-1} \\
& \cdot \lim _{r \rightarrow 1} \mathcal{E}\left(1-r^{2}\right) I f\left(r e^{i \sigma_{K}}\right) \geqq 0,
\end{aligned}
$$

for each negative points $e^{i \delta_{K}}, K=1, \cdots, 2 p-m$,

$$
\begin{array}{rl}
\lim _{r \rightarrow 1} & E(1-r) R F\left(r e^{i \delta_{K}}\right) \\
\quad=2^{2(m-p)+1} \prod_{i=1}^{m} \sin \frac{\sigma_{i}-\delta_{K}}{2} \prod_{j(\neq K)=1}^{2 p-m}\left(\sin \frac{\delta_{j}-\delta_{K}}{2}\right)^{-1} \lim _{r \rightarrow 1} \varepsilon I f\left(r e^{i \delta_{K}}\right) \geqq 0
\end{array}
$$

and for all the poles $e^{i \zeta},-\pi \leqq \zeta<\pi$, except for the positive and negative points also $\lim _{r \rightarrow 1} \mathcal{E}(1-r) R F\left(r e^{i \xi}\right) \geqq 0$. Thus, $F(z)$ is a member of the class $R_{2}(0,0)$.

Lemma 4. Let $u(z)$ be harmonic in $|z|<1$. Suppose that $\lim \inf _{z \rightarrow z^{\prime}} u(z) \geqq 0$ for each boundary points $z^{\prime}$ except for a finite number of boundary points $z_{1}, \cdots, z_{n}$. Let $u(z)=O\left(\left|z_{K}-z\right|^{-1}\right)$ as $z \rightarrow z_{K}, K=1, \cdots, m$. Then the finite limit

$$
\lim _{r \rightarrow 1} \int_{-\pi}^{+\pi}\left|u\left(r e^{i \phi}\right)\right| d \phi=M
$$

exists.

Proof. Let $C_{K}$ denote a circle orthogonal to the unit circle, and put its centre on the semi-straight line $\left[0, z_{K}\right]$ starting from the centre 0 of the unit circle. Denote the diametral length of $C_{K}$ by $r_{K}$. Assume that all $r_{K}, K$ $=1, \cdots, m$, are sufficiently small, and that all circles $C_{K}, K=1, \cdots, m$, have no common points to each other. Denote by $D_{K}$ a domain which consists of the unit circle and $C_{K}$, and by $L_{K}$ the boundary of $D_{K}$. Suppose that $U_{K}$ is a harmonic function in $D_{K}$ determined by the boundary values $u^{-}(z)$ in the intersection $C_{K} \cap L_{K}$, and 0 on the compiementary arc of $C_{K} \cap L_{K}$ with respect to $L_{K}$, where $u^{-}(z) \equiv \max [-u(z), 0]$. Then

$$
u^{-}(z) \leqq \sum_{K=1}^{m} U_{K}(z)
$$

in a domain formed by the unit circle and $C_{K}, K=1, \cdots, m$. 
Let $\zeta_{K}(z)=\left(z_{K}+z\right)\left(z_{K}-z\right)^{-1}$. Then $\zeta_{K}$ maps $C_{K}$ onto a circle $\left|\zeta_{K}\right|=\rho$, $\rho$ being a positive constant. Using the Nevanlinna's equality, for $\zeta_{K}=r e^{i \theta}$, $r<\rho$,

$$
U_{K}\left(z\left(\zeta_{K}\right)\right)=\frac{2 r \cos \theta}{\pi \rho} \int_{-\pi / 2}^{+\pi / 2} u^{-}\left(z\left(\rho e^{i \phi}\right)\right) \cos \phi(1+\eta(\rho, \phi)) d \phi
$$

where $\eta(\rho, \phi) \rightarrow 0$ as $\rho \rightarrow \infty$. On the other hand, from the hypothesis, $\lim \sup _{z \rightarrow z_{K}} u^{-}(z)\left|z-z_{K}\right|<\infty$. Therefore $\lim \sup _{\left|\zeta_{K}\right| \rightarrow \infty} u^{-}\left(z\left(\zeta_{K}\right)\right)\left|\zeta_{K}\right|^{-1}<\infty$, so that

$$
\mu_{K}=\liminf _{R \rightarrow \infty} \frac{1}{\pi \rho}-\int_{-\pi / 2}^{+\pi / 2} u^{-}\left(z\left(\rho e^{i \phi}\right)\right) \cos \phi d \phi
$$

is finite. This property holds for all $K, K=1, \cdots, m$. Thus from (2.8) and (2.9), we have for $z$ in $|z|<1$,

$$
u^{-}(z) \leqq 2 \sum_{K=1}^{m} \mu_{K} R \frac{z_{K}+z}{z_{K}-z} .
$$

Therefore $u(z)$ may be represented as the difference of two positive harmonic functions in $|z|<1$. Hence the finite limit (2.7) exists [9].

LEMMA 5. Let a function $f(z)$ be a member of the class $R_{2}(0,0)$ and regular in $|z|<1$. Then the following representation of $F$. and $R$. Nevanlinna [9] holds: Except for at most a countable set of $\theta,-\pi \leqq \theta<\pi$, the limit

$$
\alpha(\theta)=\lim _{r \rightarrow 1} \int_{-\pi}^{\theta} R f\left(r e^{i \phi}\right) d \phi
$$

exists; $\alpha(\theta)$ is a monotone increasing function of bounded variation; and for $|z|<1$,

$$
f(z)=\frac{1}{2 \pi} \int_{-\pi}^{+\pi} \frac{e^{i \theta}+z}{e^{i \theta}-z} d \alpha(\theta)+i I f(0) .
$$

Proof. For every $\theta,-\pi \leqq \theta<\pi, R f\left(e^{i \theta}\right) \geqq 0$ except for a finite number of the poles of $f(\boldsymbol{z})$ on $|\boldsymbol{z}|=1$, and for the poles $e^{i \theta_{K}}, K=1, \cdots, n, f(z)=O\left(\left|e^{i \theta K_{z}}\right|^{-1}\right)$ as $z \stackrel{A}{\rightarrow} e^{i \theta R}$. By Lemma 4 , an application of the F. and R. Nevanlinna representation theorem shows that $\alpha(\theta)$ of (2.12) exists and is of bounded variation and that the equation (2.13) holds. Moreover, an application of the A. Herzig's derivative theorem [5] shows that if $z$ tends to $e^{i \sigma},|\sigma| \leqq \pi$, along the Stolz's paths, the limit

$$
\lim _{z \rightarrow e^{i \sigma}}\left(e^{i \sigma}-z\right) f(z) e^{-i \sigma}=2 \mu_{\sigma}
$$

exists where $\mu_{\sigma}=(2 \pi)^{-1}\{\alpha(\sigma+0)-\alpha(\sigma-0)\}$. Consequently, from the defini- 
tion of $R_{2}(0,0), \alpha(\theta)$ is a monotone increasing function of $\theta,-\pi \leqq \theta<\pi$. Thus the proof is completed.

Lemma 6. Let $f(z)(\not \equiv 0)$ be meromorphic in $|z| \leqq 1$. Suppose that $f(z)$ is a member of the class $R_{1}(p, m)$. Denote the number of the zeros and poles of $f(z)$ in $|z|<1$ by $s$ and $t$ respectively and the number of the closed zeros and poles of $f(z)$ on $(z)=1$ by $2 s_{1}$ and $2 t_{1}$ respectively. Then

$$
m-p=s+s_{1}-t-t_{1} .
$$

In this lemma, the case where $f(z)$ is regular in $|z| \leqq 1$ and has no zero and pole on $|z|=1$ was proved in [7].

Proof. We denote the closed zeros and poles on $|z|=1$, the zeros and poles in $|z|<1$ and the positive and negative points on $|z|=1$, of $f(z)$ by the form in Lemmas 1,2 and 3 respectively. $F_{1}(z), F_{2}(z)$ and $g(z)$ denote the functions given in $(2.1),(2.3)$ and (2.4) respectively. Then the function $E(z)=\varepsilon F_{1}(z) F_{2}(z) g(z) f(z)$, $\varepsilon$ being +1 or -1 , is regular in $0<|z|<1$. Applying Lemma 1, 2 and 3,E(z) is a member of the class $R_{2}(0,0) . E(z)$ is expanded in the origin as follows: $E(z)=C_{0} z^{-q}+C_{1} z^{-q+1}+\cdots$ where $q=m-p-s$ $-s_{1}+t+t_{1}$. If we assume $q<0, E(z)$ is regular in $|z|<1$. By Lemma 5, we find

$$
E(z)=\frac{1}{2 \pi} \int_{-\pi}^{+\pi} \frac{e^{i \theta}+z}{e^{i \theta}-z} d \alpha(\theta)+i \operatorname{IE}(0),
$$

and $\alpha(\theta)$ is a monotone increasing function of $\theta,-\pi \leqq \theta<\pi$. Therefore from (2.16), $E(z) \equiv 0$. Hence $f(z) \equiv 0$. This contradicts our assumption. Thus $g \geqq 0$. On the other hand, $K(z)=1 / E(z)$ is also meromorphic on $|z|=1$ and regular in $0<|z|<1$. Again applying Lemmas 1,2 and $3, K(z)$ is a member of the class $R_{2}(0,0) . K(z)$ is expanded in the origin as follows: $K(z)=C_{0}^{\prime} z^{-z^{\prime}}$ $+C_{1}^{\prime} z^{-q^{\prime}+1}+\cdots$ where $q^{\prime}=-q$. Thus in this case we find also $q^{\prime} \geqq 0$. Therefore $q=0$.

Definition 6. A function $f(z)$ is said to belong to the class $D_{\gamma}^{\prime}(p, m)$, if the following conditions (a) and (b) are satisfied. Similarly the function $f(z)$ is said to belong to the class $D_{\gamma}^{\prime \prime}(p, m)$, if the following conditions (a) and (c) are satisfied.

(a) $f(z)$ is a member of the class $R_{1}(p, m)$.

(b) There exist $2 \gamma$ points, such that $1 \leqq \gamma \leqq[m / 2]$ and $1 \leqq \gamma \leqq 2 p-m+1$, having the relation that the difference of the two arguments among the positive points is $\pi$ or $-\pi$.

(c) There exist $2 \gamma$ points, such that $1 \leqq \gamma \leqq[(2 p-m) / 2]$ and $1 \leqq \gamma \leqq m+1$, having the relation that the difference of the two arguments among the negative points is $\pi$ or $-\pi$.

LeMma 7. Let $f(z)=\sum_{n=0}^{\infty} a_{n} z^{q+n}, q$ an integer, be regular in $0<|z|<1$, and the coefficients $a_{n}, n=1,2, \cdots$, be all real. Suppose that $-i f(z)$ is a member 
of the class $M^{\prime}(p)$. Then if the number of the positive points of -if(z) is denoted by $m$; the necessary and sufficient condition for $m$ to be even or zero is that -if(z) is a member of the class $D_{1}^{\prime}(p, m)$ or $D_{1}^{\prime \prime}(p, m)$, and if $e^{i \theta}, 0<\theta<\pi$, is $a$ rth order closed pole, $e^{-i \theta}$ is also a rth order closed pole. For the closed zero, this holds also.

Proof. Let $-i R f\left(e^{i \theta}\right)$ change sign $2 p$ times at $\theta=\tau,-\pi \leqq \tau_{1}<\tau_{2} \cdots<\tau_{2 p}$ $<\pi$. Then both values $\theta=\pi$ and $\theta=0$ are the elements of the set $\tau_{K}, K$ $=1, \cdots, 2 p$. If $e^{i \tau t}, 0<\left|\tau_{t}\right|<\pi$, is an element of the set $e^{i \tau_{K}}, K=1, \cdots, 2 p$, $e^{-i \tau_{t}}$ is also so. So that if $-\tau_{t}=\tau_{n},|t-s|$ is even. Therefore if $e^{i \tau_{t}}$ is a negative point, $e^{-i \tau t}$ is also a negative point. And if $e^{i \tau t}$ is a positive point, $e^{-i \tau t}$ is a positive point, too, because if $e^{-i \tau} t$ is a negative point, by the above argument $e^{i \tau t}$ would be a negative point. This contradicts our assumption. Hence $e^{-i \tau t}$ is a positive point. Thus if both points $e^{-i \pi}$ and $e^{i 0}$ belong to the positive points or negative points, $m$ is even or zero. If the one belongs to the positive points and the other to the negative points, $m$ is odd. Next, as the coefficient $a_{n}, n=1, \cdots$, of $f(z)$ are all real, for $|z|<1, I f(z)=-I f(\bar{z})$. Therefore, if $e^{i \theta}$ is a $\gamma$ th order closed pole distinct from $e^{i \tau_{K}}, K=1, \cdots, 2 p$, by (A) and (C) $e^{-i \theta}$ is also. On the other hand, for $|z|<1, R f(z)=R f(\bar{z})$. Hence, if $e^{i \theta}$ is a $\gamma$ th order closed pole among $e^{i \tau_{K}}, K=1, \cdots, 2 p$, by (B) and (C) $e^{-i \theta}$ is also.

\section{The main theorems.}

THEOREM 1. Let $q$ be an integer and $f(z)$ be a function meromorphic $|z|<1$ and a member of the class $R_{1}(p, m)$ having the form

$$
f(z)=\sum_{n=0}^{\infty} a_{n} z^{q+n} \quad\left(a_{0} \neq 0\right)
$$

in a vicinity of the origin. Denote the closed zeros and poles on $|z|=1$, the zeros and poles in $0<|z|<1$ and the positive and negative points on $|z|=1$, of $f(z)$ by the form given in Lemmas 1, 2 and 3 respectively. Then

$$
\begin{aligned}
m-p & =q+s+s_{1}-t-t_{1}, \\
\min _{-\pi \leqq \theta<\pi}\left|\frac{\left(e^{i \theta}-z\right)\left|a_{0}\right|^{2}}{e^{i(\theta-\alpha)} \bar{a}_{0}+z e^{-i \beta} a_{0}}\right| & \leqq\left|\frac{t(z)}{z^{q} P(z) Q(z)}\right| \\
& \leqq \max _{-\pi \leqq \theta<\pi}\left|\frac{e^{i(\theta+\alpha)} a_{0}+z e^{i \beta} \bar{a}_{0}}{e^{i \theta}-z}\right|, \\
\left|a_{n}\right| & \leqq \max _{-\pi \leqq \theta<\pi}\left|\phi_{n}(\theta)\right|, \quad n=0,1, \cdots
\end{aligned}
$$

where

$$
\alpha=\sum_{K=1}^{m+2 t_{1}} \sigma_{K}-\sum_{K=1}^{2 p-m+2 s_{1}} \delta_{K}, \quad \beta=2\left(\sum_{K=1}^{8} \arg b_{K}-\sum_{K=1}^{t} \arg d_{K}\right),
$$




$$
\begin{aligned}
& P(z)=\prod_{K=1}^{2 p-m+2 s_{1}}\left(e^{i \delta_{K}}-z\right) \prod_{K=1}^{m+2 t_{1}}\left(e^{i \sigma_{K}}-z\right)^{-1}, \\
& Q(z)=\prod_{K=1}^{s}\left(1-z / b_{K}\right)\left(1-\bar{b}_{K} z\right) \prod_{K=1}^{t}\left(1-z / d_{K}\right)^{-1}\left(1-\bar{d}_{K} z\right)^{-1} . \\
& \sum_{n=0}^{\infty} \phi_{n}(\theta) z^{q+n}=z^{q} P(z) Q(z) \frac{e^{i(\theta+\alpha)} a_{0}+z e^{i \beta} \bar{a}_{0}}{e^{i \theta}-z} \\
& \text { for }|z|<\underset{K}{\min }\left(\left|d_{K}\right|, K=1, \cdots t\right) .
\end{aligned}
$$

These inequalities of (3.3) and (3.4) are all sharp.

THEOREM 2. Let $q$ be an integer and a function

$$
f(z)=\sum_{n=0}^{\infty} a_{n} z^{q+n}
$$

be regular in $0<|z|<1$. Suppose that $f(z)$ is a member of the class $R_{1}(p, m)$. Denote the closed zeros and poles on $|z|=1$ and the positive and negative points of $f(z)$ by the form given in Lemmas 1 and 3 respectively. Then

$$
\begin{aligned}
m-p \geqq q+s_{1}-t_{1}, & \geqq \\
|f(z)| \leqq \max _{-\pi \leqq \theta<\pi}|W(\theta)|, & n=0,1, \ldots
\end{aligned}
$$

where

$$
\begin{aligned}
W(\theta)= & \left\{\left(A_{0} e^{i \theta}+\overline{A_{0} z}\right)\left(e^{i \theta}-z\right)^{-1}+\sum_{K=1}^{m+t_{1}-p-q-s_{1}}\left(A_{-K} z^{-K}-\bar{A}_{-K} z^{K}\right)\right\} \\
& \cdot e^{i \alpha / 2} z^{m+t_{1}-p-q-s_{1}} P(z)=\sum_{n=0}^{\infty} \psi_{n}(\theta) z^{q+n}
\end{aligned}
$$

and the $A_{-K}, K=0, \cdots, m+t_{1}-p-q-s_{1}$ are given by

$$
e^{-i \alpha / 2} z^{p+s 1-m-t_{1}} f(z) \frac{1}{P(z)}=\sum_{n=p+q+s_{1}-m-t_{1}}^{\infty} A_{n} z^{n} .
$$

These inequalities of (3.6) and (3.7) are all sharp in all the variables $a_{K}, K$ $=1, \cdots, m+t_{1}-p-q-s_{1}$.

Proof of Theorem 1. Let $F_{1}(z), F_{2}(z)$ and $g(z)$ be the functions given in (2.1), (2.3) and (2.4) respectively. Then by Lemmas 1, 2 and 3

$$
E(z)=\varepsilon F_{1}(z) F_{2}(z) g(z) f(z)
$$

is a member of the class $R_{2}(0,0)$ and $E(z)$ is expanded in the origin as follows: 


$$
E(z)=C_{0} z^{p+q+s+s_{1}-m-t-t_{1}}+\cdots \cdot
$$

By applying Lemma $6, p+q+s+s_{1}-m-t-t_{1}=0$. Hence $E(z)$ is regular in $|z|<1$. By Lemma 5 we get the representation of the form (2.13) of $E(z)$. Therefore if $R E(0)=\int_{-\pi}^{+\pi} d \alpha(\theta) \neq 0$,

$$
f(z)=\frac{1}{2 \pi R E(0)} \int_{-\pi}^{+\pi} \frac{e^{i \theta} E(0)+z \operatorname{conj} E(0)}{\left(e^{i \theta}-z\right) E F_{1}(z) F_{2}(z) g(z)} d \alpha(\theta)
$$

where $\alpha(\theta)$ is a monotone increasing function of the bounded variation. If $R E(0)=0, E(z) \equiv i I E(0)$. Hence from (3.9)

$$
f(z) \equiv i \varepsilon I E(0)\left\{F_{1}(z) F_{2}(z) g(z)\right\}^{-1} .
$$

Thus from (3.11) and (3.12), the bounds of the right-hand side of (3.3) and (3.4) hold. Now the function $K(z)=1 / E(z)$ is regular in $|z|<1$ and $K(z)$ is a member of the class $R_{2}(0,0)$, too. Therefore, using the above method, the bound of the left-hand side of (3.3) holds.

Denote the values of $\theta$ which give the maximum moduli in (3.3) and (3.4), and the minimum modulus in (3.3) by $\theta_{0}, \theta_{n}$ and $\theta_{0}^{*}$ respectively. Then the equality signs of the right and left hand of (3.3) and (3.4) are attained by the functions

$$
K_{1}^{0}(z)=z^{q} P(z) Q(z) \frac{e^{i\left(\alpha+\theta_{0}\right)} a_{0}+z e^{i \beta} \bar{a}_{0}}{e^{i \theta}-z}, \quad K_{n}(z)=z^{q} P(z) Q(z) \frac{e^{i\left(\alpha+\theta_{n}\right)} a_{0}+z e^{i \beta} \bar{a}_{0}}{e^{i \theta_{n}}-Z}
$$

and

$$
K_{2}^{0}(z)=z^{a} P(z) Q(z) \frac{\left(e^{i \theta_{0}^{*}}-z\right)\left|a_{0}\right|^{2}}{e^{i\left(\alpha-\theta_{0}^{*}\right)} \bar{a}_{0}+z e^{-i \beta} a_{0}}
$$

respectively.

Proof of Theorem 2. The function $G(z)=F_{1}(z) g(z) f(z)$ is a member of the class $R_{2}(0,0)$ and is expanded in the origin as follows:

$$
G(z)=\sum_{n=-m-t_{1}+p+q+s_{1}}^{\infty} A_{n} z^{n} .
$$

Let $f(z) \not \equiv 0$. Then by Lemma 6 , we find $m+t_{1}-p-q-s_{1} \geqq 0$. If $m+t_{1}-p$ $-q-s_{1} \neq 0$, we may write

$$
G(z)=\sum_{n=1}^{m+t_{1}-p-q-s_{1}}\left(A_{-n} z^{-n}-\bar{A}_{-n} z^{n}\right)+A_{0}+\sum_{n=1}^{\cdot m+t_{1}-p-q-s_{1}}\left(A_{n}+\bar{A}_{-n}\right) z^{n}
$$

$$
+\sum_{n=m+t_{1}-p-q-s:+1}^{\infty} A_{n} z^{n} .
$$

Define 


$$
G^{*}(z)= \begin{cases}G(z)-\sum_{n=1}^{m+t_{1}-p-q-s_{1}}\left(A_{-n} z^{-n}-\bar{A}_{-n} z^{n}\right) \\ \\ \text { if } m+t_{1}-p-q-s_{1} \neq 0, \\ \text { if } m+t_{1}-p-q-s_{1}=0 .\end{cases}
$$

Then $G^{*}(z)$ is regular in $|z|<1$ and a member of the class $R_{2}(0,0)$. Therefore by Lemma 5 , we find the representation of the form $(2.13)$ of $G^{*}(z)$. If $R A_{0}=(2 \pi)^{-1} \int_{-\pi}^{+\pi} d \beta(\theta) \neq 0$, from (3.15) we get

$$
\begin{aligned}
G(z)= & \frac{1}{2 \pi R A_{0}} \int_{-\pi}^{+\pi}\left\{\frac{e^{i \theta} A_{0}+z \bar{A}_{0}}{e^{i \theta}-z}\right. \\
& \left.+\sum_{n=1}^{m+t_{1}-p-q-s 1}\left(A_{-n} z^{-n}-\bar{A}_{-n} z^{n}\right)\right\} d \beta(\theta) .
\end{aligned}
$$

If $R A_{0}=0, G^{*}(z) \equiv i I A_{0}$. Hence

$$
G(z) \equiv i I A_{0}+\sum_{n=1}^{m+t_{1}-p-q-\varepsilon_{1}}\left(A_{-n} z^{-n}-\bar{A}_{-n} z^{n}\right) .
$$

Thus from (3.16) and (3.17), the bounds (3.6) and (3.7) hold. Denote the values of $\theta$ which give the maximum moduli of $W(\theta)$ and $\psi_{n}(\theta)$ by $\theta_{0}$ and $\theta_{n}$ respectively. Then the functions $W=W\left(\theta_{0}\right)$ and $W=W\left(\theta_{n}\right)$ give the equality signs of (3.6) and (3.7) respectively.

From the proof of Theorem 2, we easily prove the following theorems.

THEOREM 2. . In the Theorem 2, $s_{1}=0$ if the hypothesis of the closed zeros is not given.

Theorem 2 . In the Theorem 2, $s_{1}=0$ and $m=2 p$ if the hypotheses of the closed zeros and the distinction of the positive and negative points are not given and if $\sigma_{1}, \cdots, \sigma_{2 p}$ are the values of $\theta$ at which $R f(z)$ on $|z|=1$ changes sign $2 p$ times.

4. Corollaries of Theorem 1. Directly from Theorem 1 we can obtain the following corollaries.

COROLlaRY 1. Let $q$ be an integer and $f(z)$ of the form (3.1) in a vicinity of the origin be meromorphic in $|z| \leqq 1$. Suppose that $f(z)$ is a member of the class $M(p)$. Denote the zeros and poles of $f(z)$ in $0<|z|<1$ by $b_{1}, b_{2}, \cdots, b_{\text {s }}$ and $d_{1}, d_{2}, \cdots, d_{t}$ respectively, and the number of the closed zeros and poles of $f(z)$ on $|z|=1$ by $2 s_{1}$ and $2 t_{1}$ respectively. Then

$$
\begin{aligned}
0 & \leqq p+q+s+s_{1}-t-t_{1} \leqq 2 p, \\
\left|a_{n}\right| & \leqq A_{n}, \\
H(-r) & \leqq\left|f\left(r e^{i \theta}\right)\right| \leqq H(r)
\end{aligned} \quad n=0,1, \cdots,
$$


where

$$
\begin{aligned}
P_{1}(z)= & \prod_{K=1}^{s}\left(1+z /\left|b_{K}\right|\right)\left(1+z\left|b_{K}\right|\right) \\
& \cdot \prod_{K=1}^{t}\left(1-z /\left|d_{K}\right|\right)^{-1}\left(1-z\left|d_{K}\right|\right)^{-1} \\
H(z)= & \left|a_{0}\right| z^{q}(1+z)^{p+s 1+t+t_{1}-q-s+1}(1-z)^{t-p-q-s_{-s}-t_{1}-1} P_{1}(z) \\
= & \sum_{n=0}^{\infty} A_{n} z^{q+n} \text { for }|z|<\min \left[d_{1}, \cdots, d_{t}\right] .
\end{aligned}
$$

These inequalities of (4.2) and (4.3) are all sharp.

In Corollary 1 , the case where $f(z)$ is regular for $|z| \leqq 1$ and $p=q$ is due to M. S. Robertson [12]. Moreover, the case where $f(z)$ has no zero and pole on $|z|=1$ was proved by the author [7].

Proof. If the number of the positive points is denoted by $m$, by Theorem 1 , $m-p=q+s+s_{1}-t-t_{1}$. On the other hand, $2 p \geqq m \geqq 0$. Thus (4.1) holds. The bound (4.3) is obvious. For the function of Theorem 1, we have a majorant series

$$
z^{q} P(z) Q(z)\left\{e^{i(\alpha+\theta)} a_{0}+z e^{i \beta} \bar{a}_{0}\right\}\left(e^{i \theta}-z\right)^{-1} \ll H(z) .
$$

Therefore from (3.4), the bound (4.2) holds.

The sharpness of the bounds (4.2) and (4.3) is shown by the existence of the extremal function. Namely, if in the integrand in (3.11), we assume $\sigma_{K}=0, K=1, \cdots, m+2 t_{1}, \delta_{K}=-\pi, K=1, \cdots, 2 p-m+2 s_{1}, b_{K}=-\left|b_{K}\right|$, $K=1, \cdots, s, d_{K}=\left|d_{K}\right|, K=1, \cdots, t, \theta=0$ and $a_{0}=\epsilon\left|a_{0}\right|$ where $\epsilon$ denotes 1 when $m$ is even and $i$ when odd, we have an extremal function

$$
W=a_{0} z^{q} Q(Z)(1+z)^{2 p-m+2 s 1+1}(1-z)^{-\left(m+2 t_{1}+1\right)}
$$

where $b_{K}=-\left|b_{K}\right|, K=1, \cdots, s, d_{K}=\left|d_{K}\right|, K=1, \cdots, t$ and $a_{0}=\epsilon\left|a_{0}\right|$.

COROLLARY 2. Let $q$ be an integer and $f(z)$ of the form of (3.1) in a vicinity of the origin be meromorphic in $|z| \leqq 1$. Suppose that - if $(z)$ is a member of the class $D_{\gamma}^{\prime}(p, m)$. Denote the zeros and poles in $0<|z|<1$ and the number of the closed zeros and poles on $|z|=1$, of $f(z)$ by the form of Corollary 1 respectively. Then

$$
\begin{aligned}
& 0 \leqq p+q+s+s_{1}-t-t_{1} \leqq 2 p, \\
& \left|a_{n}\right| \leqq B_{n}, \quad n=0,1, \cdots, \\
& Q(-r)\left(1-r^{2}\right)^{r}\left(1+r^{2}\right)^{-\gamma} \leqq\left|f\left(r e^{i \theta}\right)\right| \leqq Q(r)
\end{aligned}
$$

where

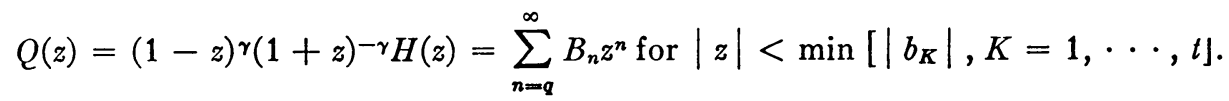


The inequalities of (4.8) and the right hand of (4.9) are sharp.

CoRollaRy 3. In Corollary 2, $Q(-r) \leqq\left|f\left(r e^{i \theta}\right)\right| \leqq\left(1+r^{2}\right)^{\gamma}\left(1-r^{2}\right)^{-\gamma} Q(r)$ and this left-hand inequality is sharp if - if $(z)$ is a member of the class $D_{\gamma}^{\prime \prime}(p, \dot{m})$ in places of the class $D_{\gamma}^{\prime}(p, m)$.

The result of a function of the class $T(p)$ by Goodman [3] is strictly stated concerning meromorphic functions in $|z| \leqq 1$ as follows:

CoROLlaRY 4. Let $q$ be an integer and $f(z)$ of the form (3.1) in a vicinity of the origin be meromorphic in $|z| \leqq 1$ and the coefficients $a_{n}, n=0,1, \cdots, b e$ all real. Suppose that -if(z) is a member of the class $M^{\prime}(p)$. Denote the zeros and poles in $0<|z|<1$ and the number of the closed zeros and poles on $|z|=1$, of $f(z)$ by the form in Corollary 1 respectively. Define $T$ by $T=p-q-s+s_{1}$ $+t+t_{1}$. Then

$$
2 p \geqq T-2 s_{1} \geqq 0,
$$

and if $2 p-T+2 s_{1}+2 t_{1} \neq 0$ and we write $m=[(T+1) / 2]$,

$$
\left|a_{n}\right| \leqq A_{n}, \quad n=0,1, \ldots
$$

where

$$
\begin{aligned}
P(z) & =\left|a_{0}\right| z^{q}(1-z)^{-2(q+o+m-t)}(1+z)^{2 m} P_{1}(z) \\
& =\sum_{n=0}^{\infty} A_{n} z^{q+n} \text { for }|z|<\min \left[\left|d_{i}\right|, i=1, \cdots, t\right] .
\end{aligned}
$$

This inequality of (4.11) is sharp.

Proof. By (3.2) in Theorem 1, (4.10) is obvious. And by Lemma 7 and Corollary 1 , it is required to prove only the classes $D_{1}^{\prime}(p, m)$ and $D_{1}^{\prime \prime}(p, m)$. Now let $-i f(z)$ be a member of the class $D_{1}^{\prime \prime}(p, m)$. Then both points $e^{-i \pi}$ and $e^{i 0}$ are negative. If $e^{i \sigma}, 0<|\sigma|<\pi$, is a positive point or a closed pole, $e^{-i \sigma}$ is also a positive point or a closed pole respectively. So that we denote two positive points or closed poles by $e^{i \sigma}$ and $e^{-i \sigma}, 0<|\sigma|<\pi$. Then

$$
\begin{aligned}
(1- & \left.z^{2}\right)\left(e^{i \sigma}-z\right)^{-1}\left(e^{-i \sigma}-z\right)^{-1} \\
& =\left(1-z^{2}\right)\left(1+e^{-i \sigma} z+e^{-2 i \sigma} z^{2}+\cdots\right)\left(1+e^{i \sigma} z+e^{2 i \sigma} z^{2}+\cdots\right) \\
& =1+\sum_{n=1}^{\infty} 2 \cos n \sigma z^{n} \ll(1+z)(1-z)^{-1} .
\end{aligned}
$$

Thus by applying Theorem 1, (4.11) holds. For a function -if(z) of the class $D_{1}^{\prime}(p, m)$ the proof of the bound (4.11) is obvious from Corollary 2. The sharpness of the bounds (4.11) is proved by the same method as in Corollary 1. 
5. Applications of Theorem 22. The following lemmas will be useful.

Lemma 8. Let $\left|a_{0}\right|,\left|a_{1}\right|, \cdots,\left|a_{p}\right|$ be any set of non-negative numbers. Then there is a unique function $F(z)$ such that

$$
F(z)=\sum_{n=0}^{\infty} a_{n} z^{n}=\left\{A_{0}(1-z)^{-1}+\cdots+A_{p} z^{p}(1-z)^{-2 p-1}\right\}(1+z)
$$

where

$$
a_{K}=(-1)^{p-K}\left|a_{K}\right|, \quad K=0, \cdots, p,
$$

and for this function

$$
a_{n}=\sum_{K=0}^{p} \Delta(p, K, n)\left|a_{K}\right|, \quad n=p+1, p+2, \cdots
$$

where

$$
\Delta(p, K, n)=2 \prod_{v=0}^{p}\left(n^{2}-v^{2}\right) /(p+K) !(p-K) !\left(n^{2}-K^{2}\right), \quad p \geqq K \geqq 0 .
$$

Conversely if $F(z)$ is of the form (5.1) and if $a_{K}, K=0, \cdots, p$, are given in (5.2), (5.3) holds.

This lemma is due to Robertson [13].

Lemma 9. Let $\left|a_{0}\right|,\left|a_{1}\right|, \cdots,\left|a_{p}\right|$ be any set of non-negative numbers. Then there is a unique function

$$
F(z)=\sum_{n=0}^{\infty} a_{n} z^{n}=A_{0}\left(z^{p+1}+z^{p}\right)(1-z)^{-2 p-1}+\sum_{K=0}^{p} A_{-K}\left(1-z^{2 K}\right)(1-z)^{-2 p} z^{p-K}
$$

where $a_{K}=(-1)^{p-K}\left|a_{K}\right|, K=0, \cdots, p$, and for such function

$$
a_{n}=\sum_{K=0}^{p} \Delta(p, K, n)\left|a_{K}\right|, \quad n=p+1, \cdots
$$

Proof of this lemma is easily obtained by applying Lemma 8.

Lemma 10. Let $\left|a_{1}\right|, \cdots,\left|a_{p}\right|$ be any set of non-negative numbers. Then there is a unique function

$$
F(z)=\sum_{n=1}^{\infty} a_{n} z^{n}=A_{0} z^{p}(1-z)^{-2 p}+\sum_{K=1}^{p-1} A_{-K}\left(1-z^{2 K}\right)(1-z)^{-2 p+1}(1+z)^{-1} z^{p-K}
$$

where $a_{K}=(-1)^{p-K}\left|a_{K}\right|, K=1, \cdots, p$, and for such function

$$
a_{K}=\sum_{K=1}^{p} D(p, K, n)\left|a_{K}\right|, \quad K=p+1, \cdots
$$

where 
(5.5) $D(p, K, n)=2 K(n+p) ! /(p+K) !(p-K) !(n-p-1) !\left(n^{2}-K^{2}\right)$.

The proof of this lemma is easily obtained by use of the lemma by Goodman and Robertson [4].

The theorems by Robertson [13] and Goodman and Robertson [4] are generalized respectively as follows:

THEOREM 3. Let a function

$$
f(z)=\sum_{n=-\infty}^{\infty} a_{n} z^{n}
$$

be regular in $0 \leqq \rho \leqq|z|<1$. Suppose that $f(z)$ is a member of the class $M(p)$. Denote the number of the closed poles of $f(z)$ on $|z|=1$ by $2 t$. Then for every $n>p+t$,

$$
\left|a_{n}+\bar{a}_{-n}\right| \leqq \sum_{K=1}^{p+\ell} \Delta(p, K, n)\left|a_{K}+\bar{a}_{-K}\right|+\frac{1}{2} \Delta(p, 0, n)\left|a_{0}+\bar{a}_{0}\right|,
$$

and this inequality is sharp in all the variables $\left|a_{K}+\bar{a}_{K}\right|, K=0, \cdots, m+t$.

THEOREM 4. Let a function

$$
f(z)=\sum_{n=1}^{\infty} a_{n} z^{n}
$$

be regular in $|z|<1$ and the coefficients $a_{n}, n=1,2, \cdots$ be all real. Suppose that - if $(z)$ is a member of the class $M^{\prime}(p)$. Denote the number of the closed poles on $|z|=1$ of $f(z)$ by $2 t$. Then for every $n>p+t$,

$$
\left|a_{n}\right| \geqq \sum_{K=1}^{p+t} D(p, K, n)\left|a_{K}\right|,
$$

and this bound is sharp in all the variables $\left|a_{K}\right|, K=1, \cdots, m+t$.

Proof of Theorem 3. $G(z)$ defined by

$$
G(z)=f(z)-i I a_{0}-\sum_{n=1}^{\infty}\left(a_{-n} z^{-n}-\bar{a}_{-n} z^{n}\right)
$$

is regular in $|z|<1$. The series of the right-hand side of (5.10) is regular on $|z|=1$ and its real part is identically zero. Hence $G(z)$ is a member of the class $M(p)$ and has $2 t$ closed poles on $|z|=1$. Therefore we need to prove the case where $a_{-K} \equiv 0, K=1,2, \cdots$, in Theorem 3. For the particular case $p+t=0$, Theorem $2_{2}$ gives

$$
f(z) \ll\left|a_{0}\right|(1+z)(1-z)^{-1} .
$$

Thus in this case (5.7) holds. When $p+t \geqq 1$, both functions $F(z)$ and $E(z)$ defined by 


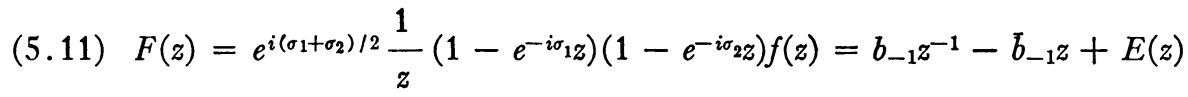

are members of the class $M(p-1)$ with $2 t$ closed poles on $|z|=1$ or a member of the class $M(p)$ with $2(t-1)$ closed poles on $|z|=1$ in accordance as the, above points $e^{i \sigma_{1}}$ and $e^{i \sigma_{2}}$ belong to the positive points or the closed poles respectively. Directly from (5.11) we find

$$
\begin{aligned}
f(z)=\sum_{n=0}^{p+t-2} a_{n} z^{n}+ & \left\{a_{p+t-1} z^{p+t-1}+\left(a_{p+t}-\left(e^{-i \sigma_{1}}+e^{-i \sigma_{2}}\right) a_{p+t-1}\right) z^{p+t}\right. \\
& \left.+\sum_{n=p+t}^{\infty} e^{-i\left(\sigma_{1}+\sigma_{2}\right) / 2} b_{n} z^{n+1}\right\}\left(1-e^{-i \sigma_{1}} z\right)^{-1}\left(1-e^{-i \sigma_{2} z}\right)^{-1}
\end{aligned}
$$

where $\sum_{n=0}^{p+t-2} a_{n} z^{n} \equiv 0$ when $p+t=1$ for convenience. On the other hand,

$$
\begin{aligned}
a_{p+t-1} z^{p+t-1}\left\{1-\left(e^{-i \sigma_{1}}\right.\right. & \left.\left.+e^{-i \sigma_{2}}\right) z\right\}\left(1-e^{\left.-i \sigma_{1} z\right)^{-1}}\left(1-e^{-i \sigma_{2} z}\right)^{-1}\right. \\
& \ll\left|a_{p+t-1}\right|\left\{\sum_{n=p+t-1}^{\infty}(n-p-t) z^{n}+z^{p+t-1}\right\} .
\end{aligned}
$$

Thus from (5.12) and (5.13), we find for $n=p+t+1, p+t+2, \cdots$,

$$
\left|a_{n}\right| \leqq\left|a_{p+t-1}\right|(n-p-t)+\left|a_{p+t}\right|(n-p-t+1)+\sum_{K=p+t}^{n-1}\left|b_{K}\right|(n-K) .
$$

Thus by applying Lemma 8 and using the induction introduced by Goodman and Robertson [4], (5.7) holds.

Applying Theorem 2 , we get an extremal function such that

$$
W=\left\{A_{0}(1+z)(1-z)^{-1}+\sum_{K=1}^{p+t} A_{-K}\left(1-z^{2 K}\right) z^{-K}\right\} z^{p+t}(1-z)^{-2(p+t)}
$$

where

$$
\begin{aligned}
a_{K} & =(-1)^{p+t-K}\left|a_{K}\right|, & K & =0, \cdots, p+t, \\
A_{-K} & =\sum_{s=0}^{p+t-K}(-1)^{s} \frac{\{2(p+t)\} !}{\{2(p+t)-s\} ! s !} a_{p+t-K-s}, & K & =0, \cdots, p+t .
\end{aligned}
$$

Hence applying Lemma 9, we get the sharpness of the bound (5.7).

By applying the lemma by Goodman and Robertson [4] and Lemma 10 and using the same method as that of Theorem 3, we obtain the proof of Theorem 4.

6. Applications of Theorem 2 and $2_{1}$. Let

$$
F(z)=\sum_{n=0}^{\infty} a_{n} z^{n}=\left\{A_{q}(1-z)^{-2 q-1}+\cdots+A_{p} z^{p-q}(1-z)^{-2 p-1}\right\}(1+z)^{2 q+1}
$$

where $p \geqq q \geqq 0$ and $a_{K}=(-1)^{p+q-K}\left|a_{K}\right|, K=0, \cdots, p-q$. And let 


$$
\Delta_{1}(p, q, K, n)=\sum_{t=0}^{2 q} \sum_{s=K}^{p-q} \Delta(p, q+s, q-t+n){ }_{2 q} C_{t 2 q} H_{s-K} .
$$

Then for every $n>p+q, a_{n}=\sum_{k=0}^{p-q} \Delta_{1}(p, q, K, n)\left|a_{K}\right|$. Similarly, let $F(z)=\sum_{n=0}^{\infty} a_{n} z^{n}=\left\{A_{q}(1-z)^{-2(q+1)}+\cdots+A_{p} z^{p-q}(1-z)^{-2(p+1)}\right\}(1+z)^{2(q+1)}$ where $p \geqq q \geqq 0$ and $a_{K}=(-1)^{p-q-K}\left|a_{K}\right|, K=0, \cdots, p-q$. And let

$$
\Delta_{2}(p, q, K, n)=\sum_{t=0}^{2 q+1} \sum_{s=K}^{p-q} \Delta_{1}(p, q+s, q+n-t)_{2 q+1} C_{t 2 q+1} H_{s-K}
$$

where

$$
\begin{array}{r}
\Delta_{1}(p, K, n)=(2 n+1) \prod_{v(\neq K)=0}^{p}(n+v+1)(n-v) /(p+1+K) !(p-K) !, \\
p \geqq K \geqq 0 .
\end{array}
$$

Then for every $n>p+q, a_{n}=\sum_{K=0}^{p-a} \Delta_{2}(p, q, K, n)\left|a_{K}\right|$. Applying Theorem 3, we easily obtain:

TheOREM 5. In addition to the hypotheses of Theorem 3, denote the number of the zeros of $f(z)$ in $|z|<1$ by s. Then $p \geqq s-t$ and for every $n>p+t$,

$$
\left|a_{n}+\bar{a}_{-n}\right| \leqq \sum_{K=1}^{8} \Delta^{*}(K, n)\left|a_{K}+\bar{a}_{-K}\right|+\frac{1}{2} \Delta^{*}(0, n)\left|a_{0}+\bar{a}_{0}\right|
$$

where

$$
\Delta^{*}(K, n)=\left\{\begin{array}{l}
\Delta_{1}\left(\frac{p+s+t}{2}, \frac{p-s+t}{2}, K, n\right), \quad p+s+t \text { even or zero, } \\
\Delta_{2}\left(\frac{p+s+t-1}{2}, \frac{p-s+t+1}{2}, K, n\right), \quad p+s+t \text { odd } .
\end{array}\right.
$$

This bound (6.1) is sharp in all the variables $\left|a_{K}+\bar{a}_{-K}\right|, K=0, \cdots, s$. Let

$$
F(z)=\sum_{n=1}^{\infty} a_{n} z^{n}=\left\{A_{q} z(1-z)^{-2(q+1)}+\cdots+A_{p} z^{p-q}(1-z)^{-2(p+1)}\right\}(1+z)^{2 p}
$$

where $p \geqq q \geqq 0$ and $a_{K}=(-1)^{p-q-K}\left|a_{K}\right|, K=q, \cdots, p$, and let

$$
D_{1}(p, q, K, n)=\sum_{t=0}^{2 q} \sum_{s=K}^{p-q} D(p, q+s, q+n-t)_{2 q} C_{t}{ }_{2 q} H_{s-K} .
$$

Then for every $n>p+q, a_{n}=\sum_{K=1}^{p-q} D_{1}(p, q, K, n)\left|a_{K}\right|$. 
TheOREM 6. In addition to the hypotheses of Theorem 4, denote the number of the closed zeros on $|z|=1$ and the number of the zeros in $|z|<1$, of $f(z)$ by $2 s_{1}$ and $s$ respectively. Then $p \geqq s+s_{1}-t$ for every $n>p+s_{1}+t$,

$$
\left|a_{n}\right| \leqq \sum_{K=1}^{\infty} D^{*}(K, n)\left|a_{K}\right|
$$

where

$$
D^{*}(K, n)=\left\{\begin{array}{l}
\Delta_{1}\left(\frac{p+s+s_{1}+t}{2}, \frac{p-s+s_{1}+t}{2}, K, n\right), p+s+s_{1}+t \text { odd }, \\
D_{1}\left(\frac{p+s+s_{1}+t}{2}, \frac{p-s+s_{1}+t}{2}, K, n\right), p+s+s_{1}+t \text { even. }
\end{array}\right.
$$

This inequality (6.2) is sharp in all the variables $\left|a_{K}\right|, K=1, \cdots, s$.

In Theorem $6, s_{1}=0$ if the hypothesis of the closed zero is not given.

Proof of Theorem 6. Let $f(z) \equiv 0$. Denote the number of the positive points by $m$. Then by Lemma $6, m-p=s+s_{1}-t$. When $m$ is odd, (6.2) holds by Theorem 5. When $m$ is even or zero, $-i f(z)$ is a member of the class $D_{1}^{\prime}(p, m)$ or $D_{1}^{\prime \prime}(p, m)$ by Lemma 7 . By applying the following lemma we easily see the bound (6.2).

LemMA 11. Let $\left|a_{0}\right|,\left|a_{1}\right|, \cdots,\left|a_{p-1}\right|$ be any set of non-negative numbers. Then there is a unique function $F(z)$ such that

$$
F(z)=\sum_{n=0}^{\infty} a_{n} z^{n}=\left\{A_{1}(1-z)^{-2}+\cdots+A_{p} z^{p-1}(1-z)^{-2 p}\right\}(1+z)^{2}
$$

where for $K=0, \cdots, p-1$,

$$
a_{K}=(-1)^{p-1-K}\left|a_{K}\right|,
$$

and for this function

$$
a_{n}=\sum_{K=0}^{p-1} D_{1}(p, 1, K, n)\left|a_{K}\right|, \quad n=p+1, \cdots
$$

Conversely if $F(z)$ is of the form (6.3) and if $a_{K}, K=0, \cdots, p-1$, are given in (6.4), (6.5) holds.

The sharpness of the bound (6.2) is given by applying Theorem 2 and Lemma 10.

7. Remark. By virtue of the idea based on the existence of the diametral line used by N. G. de Bruijin [1], S. Ozaki [10], and S. Ozaki, T. Takatsuka and $\mathrm{T}$. Umezawa [11], the set of regular functions for $|z| \leqq 1$ of the class $D_{1}^{\prime}(p, 2 p)$ contains the set of the multivalently star-shaped functions of order $p$ for $|z| \leqq 1$ with respect to the origin. Therefore in Corollary 2, the 
part with respect to the set $S(p)$ with assigned zeros in $|z|<1$ is due to Good$\operatorname{man}[2]$.

The set of functions which satisfies the hypothesis of Theorem 4 implies the set of functions which satisfies the hypothesis of Theorem 6, and the bounds (5.9) and (6.2) are sharp respectively. Therefore we find for all $s$, $s \leqq p+t$,

$$
\sum_{K=0}^{s} D^{*}(K, n)\left|a_{K}\right| \leqq \sum_{K=0}^{p+t} D(p, K, n)\left|a_{K}\right|, \quad n=p+t+1, \cdots
$$

Similarly, we get for all $s, s \leqq p+t$

$$
\sum_{K=0}^{s} \Delta^{*}(K, n)\left|a_{K}\right| \leqq \sum_{K=0}^{p+t} \Delta(p, K, n)\left|a_{K}\right|, \quad n=p+t+1, \cdots .
$$

The equality signs in (7.1) and (7.2) hold when $s=p+t$. From Theorem 6 we have immediately the following theorem.

THEOREM 7. Let $f(z)$ given by (5.8) be p-valent starlike with respect to a point on the real axis, for $|z| \leqq 1$. Suppose that all the coefficients of $f(z)$ are all real. Denote the number of the zero points of $f(z)$ in $|z|<1$ by s. Then the bound (6.2) holds where

$$
D^{*}(K, n)= \begin{cases}\Delta_{1}\left(\frac{p+s}{2}, \frac{p-s}{2}, K, n\right), & p+s \text { odd }, \\ D_{1}\left(\frac{p+s}{2}, \frac{p-s}{2}, K, n\right), & p+s \text { even. }\end{cases}
$$

The $p$-valent starlike function with respect to a point has been studied previously by Robertson and others, and from (7.1) this Theorem 7, in some sense, precises the theorem by Goodman and Robertson in [4, Theorem 3].

\section{REFERENCES}

1. N. G. de Bruijn, Ein Satz über schlichte Funktionen, Nederl. Akad. Wetensch. vol. 44 (1941) pp. 47-49.

2. A. W. Goodman, On the Schwarz-Christoffel transformation and p-valent functions, Trans. Amer. Math. Soc. vol. 68 (1950) pp. 204-223.

3. - Typically-real functions with assigned zeros, Proc. Amer. Math. Soc. vol. 2 (1951) pp. 349-357.

4. A. W. Goodman and M. S. Robertson, A class of multivalent functions, Trans. Amer. Math. Soc. vol. 70 (1951) pp. 127-136.

5. A. Herzig, Die Winkelderivierte und des Poisson-Stieltzes-Integral, Math. Z. vol. 46 (1940) pp. 129-156.

6. J. Itô, On the function whose imaginary part on the unit circle changes its sign $2 p$ times, Sci. Rep. Tokyo Bunrika Daigaku sect. A vol. 4 (1944) pp. 107-114.

7. - On the function whose real part on the unit circle changes its sign finite times, Bull. Nagoya Inst. Tech. vol. 3 (1951) pp. 293-305. 
8. S. Kakeya, On the function whose imaginary part on the unit circle changes its sign only twice, Proc. Japan Acad. vol. 18 (1942) pp. 435-439.

9. R. Nevanlinna, Eindeutige analytische Funktionen, Berlin, 1936.

10. S. Ozaki, On the theory of multivalent functions, Sci. Rép. Tokyo Bunrika Daigaku sect. A vol. 4 (1941) pp. 45-87.

11. S. Ozaki, T. Takatsuka and T. Umezawa, Analytic functions starlike of order $P$ in one direction, J. Dept. Educ. Shizuoka Univ. vol. 1 (1950) pp. 81-87.

12. M. S. Robertson, $A$ representation of all analytic functions in terms of functions with positive real parts, Ann. of Math. vol. 38 (1937) pp. 770-783.

13. - A coefficient problem for functions regular in an annulus, Canad. J. Math. vol. 4 (1952) pp. 407-423.

Nagoya Institute of Technology, NAGOYa, JaPAN 